\title{
A relevância da aplicação prévia de formulários para o conhecimento do público- alvo, no contexto de aulas on-line promovidas por uma liga acadêmica de saúde coletiva
}

The relevance of the prior application of forms for the knowledge of the target audience, in the context of online classes promoted by an academic collective health league

La relevancia de la aplicación previa de formularios para el conocimiento del público objetivo, en el contexto de las clases online impulsadas por una liga académica colectiva de salud

Rafaela Victoria Camara Soares ORCID: https://orcid.org/0000-0002-1412-5467 Universidade do Estado do Pará, Brasil E-mail: rafaela.vsoares@aluno.uepa.br

Láelia Anayze Ribeiro Macedo ORCID: https://orcid.org/0000-0003-3726-5689 Universidade do Estado do Pará, Brasil E-mail: macedolaelia@gmail.com Fabíola Eloise Rodrigues Dias ORCID: https://orcid.org/0000-0002-1981-3365 Universidade do Estado do Pará, Brasil E-mail: elloisedias@gmail.com

Ananda Taysa Dantas Ribeiro

ORCID: https://orcid.org/0000-0002-2433-932X Universidade do Estado do Pará, Brasil E-mail: anandantasr@gmail.com

Matheus Sallys Oliveira Silva

ORCID: https://orcid.org/0000-0003-2722-7558 Universidade do Estado do Pará, Brasil E-mail: mattheussallys@gmail.com Yasmim Silva Sousa

ORCID: https://orcid.org/0000-0002-0229-1388 Universidade do Estado do Pará, Brasil E-mail: yasmimsilva2605@gmail.com Lenise Ascenção Silva Nunes

ORCID: https://orcid.org/0000-0001-8471-1819 Universidade do Estado do Pará, Brasil E-mail: lenisenunes@ outlook.com

Juarez Rebelo de Araújo

ORCID: https://orcid.org/0000-0002-9230-2801 Universidade do Estado do Pará, Brasil E-mail: juarezrebelo@ @otmail.com

Franciane de Paula Fernandes ORCID: https://orcid.org/0000-0002-4617-1919 Universidade do Estado do Pará, Brasil E-mail: franciane.fernandes@uepa.br

Lívia de Aguiar Valentim

ORCID: https://orcid.org/0000-0003-4255-8988 Universidade do Estado do Pará, Brasil E-mail: livia.valentim@uepa.br

Sheyla Mara Silva de Oliveira

ORCID: https://orcid.org/0000-0001-6666-2363 Universidade do Estado do Pará, Brasil E-mail: sheylaoliveira@uepa.br

\section{Resumo}

Os avanços digitais têm mudado o processo ensino aprendizagem. Pensando nisto, surgiu a proposta de descrever o uso da plataforma do Google Forms como método de pesquisa e constatar sua efetividade do contato pré e pós da atividade remota. A primeira aula online da liga realizada durante o período da quarentena, obteve um total de 88 
participantes. Sendo 77 estudantes de graduação (87,5\%), 02 estudantes de pós-graduação (2,27\%), e 09 profissionais $(10,23 \%)$. Presume-se que a predominância de graduandos se deve a maior oferta de cursos de graduação do campus, uma vez que 54 ouvintes $(61,36 \%)$ pertenciam a instituição que promovia o evento. Dos participantes $(71,95 \%)$, 59 pessoas afirmaram nunca ter tido contato com a população indígena. Com isso, a aplicação desses formulários pode nortear para a real necessidade dos participantes, e a liga em seu caráter educativo promove os ambientes para o aprendizado em saúde. Concluindo que diante do cenário de pandemia em que aglomerações devem ser evitadas, no que concerne a possibilidade de explorar tópicos diversificados por meio do uso de recursos tecnológicos, essa metodologia da plataforma do Google Forms é um instrumento eficaz para planejamento de ações seja de ensino e/ou saúde.

Palavras-chave: Saúde coletiva; Ensino; Google Forms.

\begin{abstract}
Digital advances have changed the teaching-learning process. With this in mind, there was a proposal to describe the use of the Google Forms platform as a research method and verify its effectiveness in the pre and post contact of the remote activity. The league's first online class held during the quarantine period had a total of 88 participants. 77 undergraduate students (87.5\%), 02 graduate students $(2.27 \%)$, and 09 professionals $(10.23 \%)$. It is assumed that the predominance of undergraduates is due to the greater offer of undergraduate courses on campus, since 54 listeners $(61.36 \%)$ belonged to the institution that promoted the event. Of the participants $(71.95 \%)$, 59 people said they had never had contact with the indigenous population. Thus, the application of these forms can guide the real needs of the participants, and the league, in its educational character, promotes environments for learning in health. Concluding that in view of the pandemic scenario in which agglomerations should be avoided, with regard to the possibility of exploring diversified topics through the use of technological resources, this methodology of the Google Forms platform is an effective instrument for planning actions, whether teaching and /or health.
\end{abstract}

Keywords: Public health; Teaching; Google Forms.

\title{
Resumen
}

Los avances digitales han cambiado el proceso de enseñanza-aprendizaje. Con esto en mente, se propuso describir el uso de la plataforma Google Forms como método de investigación y verificar su efectividad en el pre y post contacto de la actividad remota. La primera clase en línea de la liga celebrada durante el período de cuarentena tuvo un total de 88 participantes. 77 estudiantes de pregrado (87,5\%), 02 estudiantes de posgrado (2,27\%) y 09 profesionales $(10,23 \%)$. Se asume que el predominio de los estudiantes de pregrado se debe a la mayor oferta de cursos de pregrado en el campus, ya que 54 oyentes $(61,36 \%)$ pertenecían a la institución que promovió el evento. De los participantes (71,95\%), 59 personas dijeron que nunca habían tenido contacto con la población indígena. Así, la aplicación de estos formularios puede orientar las necesidades reales de los participantes, y la liga, en su carácter educativo, promueve entornos de aprendizaje en salud. Concluyendo que ante el escenario pandémico en el que se deben evitar aglomeraciones, en cuanto a la posibilidad de explorar temas diversificados mediante el uso de recursos tecnológicos, esta metodología de la plataforma Google Forms es un instrumento eficaz para planificar acciones, ya sean docentes y / o salud.

Palabras clave: Salud pública; Docencia; Formularios de Google.

\section{Introdução}

O ensino por sua vez tem impacto importante na promoção da construção ética e responsável dos cidadãos, em seu espectro de metodologias para a difusão de conhecimento inclui-se a educação tecnológica. Os avanços digitais trouxeram um avanço considerável para a ambientação da sala de aula e auxiliaram em diversas formas o trabalho do docente. Um ensino com a utilização dessas tecnologias permite ao aluno o contato com a cultura tecnológica do seu tempo. Utilizada de forma adequada, a educação tecnológica contribui para que os alunos aprendam a buscar as informações e desenvolvam um julgamento crítico quanto ao grande volume de informação disponível na rede mundial de computadores (Mec, 2017; Silva et al, 2005; Garutti \& Ferreita, 2016; Kenski, 2008;).

Concomitante a isto, no ambiente universitário as ligas acadêmicas são estratégias fundamentais nas universidades pelo seu papel de aproximação entre a academia e a comunidade. A sua estrutura composta discentes e supervisionadas por docentes viabiliza a indissociabilidade entre o ensino, a pesquisa e a extensão, sendo elas as principais responsáveis por auxiliar os discentes a preencher as lacunas existentes na formação acadêmica (Cavalcante et al., 2018)

Com advento da pandemia causada pelo novo coronavírus (SARS-CoV-2) e a necessidade da realização de um isolamento social preconizado pelos principais órgãos de saúde mundiais, a universidade e as organizações estudantis tiveram 
que se adaptar para seguirem em funcionamento e adotar o novo "normal". Com isso a reinvenção das principais organizações administrativas foi necessária, tais como a adoção de mídias sociais na realização de atividades, que comumente eram presenciais, para a forma remota, como aconteceu com a Liga Acadêmica de Saúde Coletiva na Amazônia (LIASCOA), criada pelos discentes da Universidade do Estado do Pará, Campus XII (Santarém/PA).

Sendo esses ambientes remotos, importante ferramenta para a coleta de dados sobre o conhecimento prévio do ouvinte a um determinado assunto, assim como a efetividade da atividade remota no conhecimento deste indivíduo. Fato possibilitado pela organização existentes plataformas digitais como o Google Classroom, salas de reunião por meio do Google Meet e coleta de dados pelo Google Forms.

A coleta de dados por meio do Google Forms oferece vários caminhos para a apreciação dos dados, pois permite que autor possa compartilhar o formulário via e-mail, ou link nas redes sociais como Whatsapp, e viabiliza a quem vai responder disponibilizar a resposta de onde estiver. Os resultados da pesquisa pelo Google Forms, podem ser vistos em forma de gráficos e planilhas, proporcionando um resultado quantitativo de forma prática e organizada, facilitando a análise dos dados (Mota, 2020; Andres, et al., 2020).

Nesse sentido, este estudo tem por objetivo descrever o uso da plataforma do Google Forms como método de pesquisa. Para alcançar tal objetivo, este trabalho busca apresentar como ocorreu o uso da Plataforma Google Forms para realização compilação de dados no processo de pesquisa de aulas e constatar sua efetividade do contato pré e pós da atividade remota.

\section{Metodologia}

Trata-se de uma pesquisa quali-quantitativa, onde se busca avaliar as percepções e conhecimentos dos sujeitos, quantificando as informações para verificar as fragilidades vivenciadas com relação a temática abordada (Silva et al, 2018), onde avaliou-se a opinião pública, como assim categoriza a Resolução CNS n $n^{\circ}$ 510/16. Para tal foi utilizado a plataforma digital Google Formulários para a aplicação de dois questionários de múltipla escolha, com a intenção de compreender a opinião e os conhecimentos pré-existentes dos inscritos em dois eventos online organizados pela Liga Acadêmica de Saúde Coletiva na Amazônia - LIASCOA.

O primeiro formulário se referia ao evento realizado em dois dias, pela plataforma Zoom, intitulado "Ciclo de Aulas: Saúde Indígena em Tempos de Pandemia" ocorrido nos dias 10 e 17 de Outubro de 2020. Foi estruturado em cinco perguntas com respostas objetivas para esclarecer os pensamentos e conviç̧ões dos inscritos a respeito do atendimento as populações indígenas.

O segundo formulário refere-se ao evento online sediado na plataforma digital Google Meet, intitulado "Café Com Saúde Coletiva: Saúde Ocupacional sob a Percepção dos Profissionais Atuantes na Linha de Frente da Pandemia da COVID19" ocorrido no dia 23 de Novembro de 2020. O questionário se deu em quatro perguntas de múltipla escolha com o objetivo de elucidar os conhecimentos e opiniões no tocante a saúde ocupacional, física e mental de profissionais de saúde durante a pandemia.

\section{Resultados e Discussão}

A primeira aula online da liga realizada durante o período da quarentena, obteve um total de 88 participantes. Sendo 77 estudantes de graduação (87,5\%), 02 estudantes de pós-graduação (2,27\%), e 09 profissionais (10,23\%). Presume-se que a predominância de graduandos se deve a maior oferta de cursos de graduação do campus, uma vez que 54 ouvintes $(61,36 \%)$ pertenciam a instituição que promovia o evento. 
Alunos de outras instituições corresponderam a 32 pessoas (36,36\%), e 02 indivíduos não declararam sua instituição (2,27\%). Em relação ao aceite em responder o questionário, 82 (93,18\%) dos participantes foram colaborativos, apenas 6 $(6,82 \%)$ recusaram.

Na segunda aula houve apenas 01 recusa $(1,72 \%)$, em relação a 58 participantes, 57 (98,28\%) aceitaram responder ao questionário. Na qual, 51 indivíduos pertenciam a instituição promovedora da atividade, e 07 (12,07\%) apresentavam vínculo institucional com outras instituições.

Manteve-se o domínio da participação de estudantes de graduação 55 alunos $(94,83 \%)$ e 3 profissionais $(5,17 \%)$. Estes parâmetros possibilitam conhecer o tipo de público que tem buscado participar das aulas da liga, e influência na decisão das próximas temáticas das novas aulas.

Ademais, a prevalência de discentes de graduação da própria instituição que a liga foi criada, colabora para que a liga acadêmica invista em atividades que reduzam algumas lacunas na formação que são conhecidos também pelos seus membros, criando novos espaços para aprendizados e vivências. Sobretudo, quando se refere a um ano atípico cujo aulas foram interrompidas, campos de prática e estágios indisponíveis.

Nos cursos de saúde na Universidade do Estado do Pará, a atuação e interação dos estudantes com as populações indígenas, na qual foi a primeira temática de aula online, é trabalhada a partir da inserção da disciplina Populações Tradicionais, como disciplina obrigatória, na matriz curricular dos cursos, pois há o entendimento de que na região Norte, há uma parcela predominante de povos indígenas, na qual existe a necessidade de que atenção prioritária seja dada às questões étnicas e culturais da população nativa (IBGE, 2010).

Ainda que a academia tenha uma matriz curricular de adesão à saúde indígena percebe-se certas lacunas durante a graduação, no que diz à construção de diálogos interculturais que reconheçam e fortaleçam a produção das diferenças culturas e aprofundem a democratização das relações entre o Estado e os Povos Indígenas.

Dos participantes (71,95\%), 59 pessoas afirmaram nunca ter tido contato com a população indígena. Essa interação seja ao longo da graduação ou após a formação é essencial no âmbito da promoção de espaços de troca e diálogo entre sujeitos de tradições culturais distintas e abrir novos caminhos de reflexão no campo da saúde indígena e da saúde coletiva, para o qual é imprescindível refletir sobre nossas possibilidades de cuidado e construções de um cuidado coletivo e diferenciado (Bahia, 2018).

Além disso, pautada na opinião pública dos participantes foi percebida pouca interação e/ou interesse por trabalhar na assistência à população indígena, visto que apenas 27 pessoas (30,7\%) do total de participantes afirmou a pretensão em atuar na área, (64,63\%), 53 inscritos apresentou dúvida, afirmando que, talvez, atuaria na assistência a indígenas.

Mesmo com esses dados, dentro da atividade que foi realizada, observou-se uma adesão significativa relacionada a temática. Foi notório certa negligência relacionada a expansão de informações associadas às populações tradicionais, pois, se percebeu a pouca interação dos participantes durante a aula. Analisando o contexto geral da saúde indígena vê-se a ligação direta com a saúde coletiva, com o projeto político da Reforma Sanitária e a criação do SUS (Kabad; Pontes; Monteiro, 2020).

Com isso, as ligas acadêmicas, sobretudo, no que desrespeita a LIASCOA, tenta aprofundar o conhecimento a respeito das populações tradicionais juntamente com a universidade. Uma vez, que se faz de extrema importância na assistência em saúde da região do oeste do Pará. De forma a fortalecer ambientes através de aulas online, webinário, conferências que incentive e encoraje novos profissionais e os já atuantes a defenderem e trabalharem com a saúde indígena e quilombola.

É válido destacar a carência e até a inexistência de um sistema de informação adequado, o que impede uma análise minimamente detalhada acerca da epidemiologia e incidência de tais agravos, além de imitar as tentativas de avaliação de 
programas de controle das principais doenças (Coimbra Junior; Santos; Cardoso, 2007). Ter profissionais engajados na atenção à saúde indígena é nevrálgico, diante de tantas dificuldades para uma assistência integral dessa população.

Segundo Kabad, Pontes, Monteiro (2020), o distanciamento das pessoas relacionado a temática deve-se aos diferentes campos disciplinares, abrangendo novos grupos sociais e implementando abordagens complexas e interdisciplinares, principalmente expondo um cenário de inúmeros desafios vivenciados pelas desigualdades em saúde que afetam os povos indígenas e pelas importantes transformações dessa população em termos demográficos, epidemiológicos nutricionais e socioculturais.

Em relação aos participantes que já realizaram atendimentos com indígenas 22 indivíduos (25\%) afirmaram ter feito parte da assistência dessa população. 54,54\% (12 inscritos alegaram a atenção a complicações relacionadas a doenças infecciosas (COVID e outros), com os povos indígenas. Nesse contexto, entende-se que a formação do profissional de saúde para atuar com a população indígena deve estar pautada em uma prática social que se qualifica pela competência em saber lidar com as especificidades geográficas e culturais dos povos nativos (Pina; Püschel; Rocha; Vieira; Fonseca; Oliveira, 2016).

Para isso, os cursos da saúde por meio de seus docentes, deve implementar iniciativas sistemáticas de ensinoaprendizagem, discutindo a biodiversidade na Amazônia e, especificamente, as populações indígenas, não somente no campo da saúde, mas também na educação, no ambiente e na tecnologia (Pina; Püschel; Rocha; Vieira; Fonseca; Oliveira, 2016).

$\mathrm{Na}$ aula intitulada: Café com saúde coletiva, uma abordagem sobre saúde ocupacional sob a percepção de profissionais atuantes na linha de frente da pandemia da COVID-19. Percebeu-se que a maioria dos participantes não estavam atuando de forma direta durante a pandemia, $(31,58 \%), 38$ participantes. Porém a maioria dos ouvintes estiveram, em contato com portadores da COVID-19, 34 pessoas $(59,65 \%)$.

Em relação a esse contato, a maioria não sabia que essas pessoas estavam positivadas para o novo coronavírus. Além disso, os locais de maior exposição com mais de 38\% foram na pesquisa epidemiológica do governo do Estado do Pará em parceria com a Secretaria de saúde do Estado do Pará (SESPA) e a Universidade do Estado do Pará (UEPA) e na casa de pacientes.

Apenas 4 pessoas $(11,76 \%)$ acreditam ter se contaminado em locais sociais, os demais alegaram a contaminação em ambientes hospitalares, unidades básicas de saúde, e unidades de pronto atendimento, além da pesquisa já citada. O que faz a liga acreditar que a maior parte dos participantes são conscientes de seus papéis coletivos de isolamento e distanciamento social, uma vez que se pressupõe que os locais nas quais foram expostos são frequentados em situações de necessidade.

O contato com positivados do Sars-Cov-2 trouxe desconforto e insegurança para mais de 23\%, isso pode ocasionar consequências mentais, relacionadas a cognição e a emoção (Lima et al, 2020). Dessa forma, é necessário a prevenção de possíveis patológicas na saúde mental, pois pode afetar diretamente na qualidade de vida das pessoas.

Esse dado é fundamental para a liga, uma vez que pode influenciar nas novas atividades, a saúde mental dos acadêmicos e profissionais da saúde, também é prioridade para a saúde coletiva. Dessa forma, pensa-se em espaços que oportunizem a promoção em saúde e o autocuidado, mesmo que de forma online, esse cenário pode ser criado através de parcerias com psicólogos e ligas acadêmicas de saúde mental.

Com isso, a aplicação desses formulários motiva e pode nortear para a real necessidade dos participantes, e a liga em seu caráter educativo promove os ambientes para o aprendizado em saúde.

\section{Conclusão}

Diante da conjuntura supracitada é notório o alcance do objetivo proposto nesse estudo, tendo em vista que, através da aplicação dos formulários eletrônicos foi possível coletar, analisar e interpretar dados. Além disso, destaca-se a importância 
dessa metodologia de pesquisa, principalmente no cenário de pandemia em que aglomerações devem ser evitadas, no que concerne a possibilidade de explorar tópicos diversificados por meio do uso de recursos tecnológicos.

Sob essa perspectiva, essa tipologia de estudo permitiu, através de investigação remota, caracterizar o público participante, perceber o grau de interesse referente as proposições discutidas, bem como compreender o cenário no qual estava inserido associado as temáticas desenvolvidas. Nesse contexto, os eventos ocorridos vinculam-se a caracterização das ligas acadêmicas como potenciais agentes da educação permanente, as quais devem prosseguir, mesmo em circunstâncias adversas, com o compromisso de aprimorar os conhecimentos relacionados a temáticas amplamente debatidas, mas também àquelas negligenciadas, como a saúde indígena e ocupacional.

\section{Referências}

Andres, F. C et al. (2020). Conhecimento de enfermeiros acerca das práticas integrativas e complementares em saúde. Research, Society and Development, $9(7): 1-15$.

Bahia, L. (2018). Trinta anos de Sistema Único de Saúde (SUS): uma transição necessária, mas insuficiente. Caderno de Saúde Pública, 34(7), e00067218.

Cavalcante, A. S. P. et al. (2020). As Ligas Acadêmicas na Área da Saúde: Lacunas do Conhecimento na Produção Científica Brasileira. Revista Brasileira de Educação Médica, 42(1), 194-204. https://www.scielo.br/pdf/rbem/v42n1/0100-5502-rbem-42-01-0199.pdf.

Coimbra Junior, Carlos E. A., Santos. R. V., \& Cardoso, A. M. (2007). Processo saúde-doença. Vigilância Alimentar e Nutricional Para a Saúde Indígena, 4774.

Garutti S, \& Ferreira V. L (2016). Uso das Tecnologias de Informação e Comunicação na Educação. Revista CESUMAR - Ciências Humanas e Sociais Aplicadas, 20, 355-372.

Instituto Brasileiro de Geografia e Estatística (2012). Censo Demográfico 2010: características gerais dos indígenas. Resultados do universo. IBGE.

Lima S O et al (2020). Impactos no comportamento e na saúde mental de grupos vulneráveis em época de enfrentamento da infecção COVID-19: revisão narrativa. Revista Eletrônica Acervo Saúdee4006-e4006.

Kabad, J. F., Pontes, A. L. De M., \& Monteiro, S. (2020). Relações entre produção cientifica e políticas públicas: o caso da área da saúde dos povos indígenas no campo da saúde coletiva. Ciência \& Saúde Coletiva, 25, 1653-1666.

Kenski V. M (2008). Educação e tecnologias: o novo ritmo da informação. (3a ed.), Papirus, 148 p.

Ministério da Educação (MEC)/Secretaria de Educação Básica (2017). Política de Inovação Educação Conectada.

Mota J.S. (2019) Utilização do google forms na pesquisa acadêmica. Revista Humanidades e Inovação 6(12):372-380.

Pina, R. M. P., et al (2016). Ensino de enfermagem na saúde indígena: uma abordagem problematizadora - relato de experiência. Revista de Enfermagem, UFPE On-Line, 10, 1556-1561.

Silva, A. da, Castro-Silva, C. R., \& Moura, L. (2018). Pesquisa qualitativa em saúde: percursos e percalços da formação para pesquisadores iniciantes. Saúde e Sociedade. 27, 632-645.

Silva H. et al (2005). Inclusão digital e educação para a competência informacional: uma questão de ética e cidadania e cidadania. Ciência da Informação, Brasilia, 34, 28-36.

Teixeira, C. F. S. et al (2020). A saúde dos profissionais de saúde no enfrentamento da pandemia de Covid-19. Ciência \& Saúde Coletiva25, $3465-3474$. 